\title{
The criteria for dynamical mass transfer of the main-sequence donor stars
}

\author{
H. Ge ${ }^{1,2}$, R. F. Webbink ${ }^{3}$, X. Chen ${ }^{1,2}$ and Z. Han $^{1,2}$ \\ ${ }^{1}$ National Astronomical Observatories/Yunnan Observatory, Chinese Academy of Sciences, \\ Kunming 650011, China \\ ${ }^{2}$ Key Laboratory for the Structure and Evolution of Celestial Objects, Chinese Academy of \\ Sciences, Kunming 650011, China \\ ${ }^{3}$ Department of Astronomy, University of Illinois, 1002 W. Green St. Urbana, IL 61801, USA
}

\begin{abstract}
Mass transfer is very common in binary evolution and it dominates the evolutionary fate of binaries. Two crucial problems i.e. dynamical mass transfer and common envelope evolution, are not well understood yet. Here we focus on the first problem, and systematically show the critical mass ratio for dynamical mass transfer when the donor stars are still on the main sequence (MS).
\end{abstract}

Keywords. Binaries:close, stars:evolution, stars:mass-loss, stars:interios

\section{Introduction}

Mass transfer occurs in many different types of close binary stars, and it dominates the evolutional feature in the binary systems. When dynamical timescale mass transfer occurs, the donor star radius is driven by hydrostatic relaxation far beyond its Roche lobe radius. In this case, the binary enters a common envelope (CE) phase because the donor envelope is tidally disrupted, engulfing the companion star. Both of the onset and termination of a $\mathrm{CE}$ evolution depend on the response of the donors remnant layers and the Roche lobe to rapid/adiabatic mass loss. In general, we think that if the mass ratio ( $q=M_{\text {donor }} / M_{\text {accretor }}$ ) less than a critical value $q_{\text {crit }}$, the mass transfer will be stable against dynamical time scale.

The main sequence (MS) donor stars with mass larger than about $1.5 M_{\odot}$ have a radiative envelope, and we call them the radiative donor stars in this poster. The instabilities against dynamical mass transfer of the radiative donors are related to the progenitors of Algol-type binaries, Type Ib/Ic supernovae, short-period of binaries consisting of a compact primary and a helium/Wolf-Rayet-star companion, and double degenerates. Mass transfer tends to be stable against dynamical mass transfer for mass ratio $q \lesssim 2.14$ based on the polytropic model of full radiative donor star. We investigate the adiabatic response of the radiative donor stars via adiabatic mass loss model and find that the critical mass ratio is different from the results of the polytropic model. The difference between our results and the polytropic models may change the knowledge on the evolution and the fate of binary systems with radiative donor stars.

The mass transfer of a MS donor star with mass smaller than about $1.5 M_{\odot}$ is related to the evolution of cataclysmic variables $(\mathrm{CVs})$. There is a great deal more physics involved in understanding the evolution of CVs, i.e., the angular momentum loss caused by magnetic stellar wind or gravitational wave radiation, the accretion of the white dwarfs, the response of the donor star to rapid mass transfer. We focus on the adiabatic response of the MS donor star and explore the critical mass ratio of the low mass binary systems. 


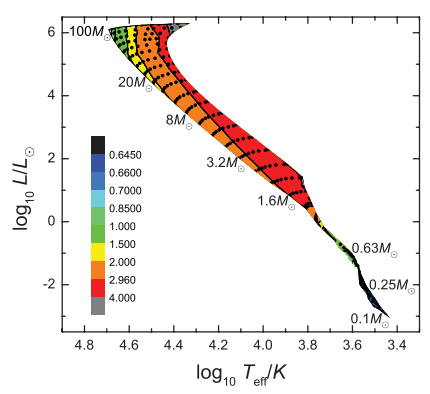

Figure 1. The contour map of the critical mass ratio $q_{\text {crit }}$,of the binary systems with main-sequence (MS) donor stars, on the Hurtzsprung-Russell diagram.

\section{Computing method}

We use the stellar evolution code built and described by Ge et al. (2010). This code solves the equations of the adiabatic mass-loss model and reproduces the response of a donor star undergoing rapid mass loss. We examine the instability of mass transfer by comparing the responses of donor star radius and Roche lobe radius to adiabatic mass loss. We use the threshold of the critical mass ratio of dynamical mass transfer in binary systems for a conserved case. The mass of these MS donor stars ranges from 0.1 to $100 M_{\odot}$, and the metallicity, $\mathrm{Z}$, is fixed to 0.02 .

\section{Results}

We summary the results of the critical mass ratio $q_{\text {crit }}$ for dynamical mass transfer as a function of location in the Hertzsprung-Russell diagram. To understand the results of the critical mass ratio, we have to keep the radial responses of different donor stars during adiabatic mass loss in mind. In radiative zones, entropy increases outwards, and the removal of high-entropy surface layers brings lower-entropy (denser) material toward the surface, leading to contraction; in convective zones, the entropy profile is nearly flat (except for super-adiabatic surface layers) and the star expands in response to mass loss (see Ge et al. 2010, for details).

Based on Fig.1., we can find that: (1) the critical mass ratio increases as the radiative donor star evolves on the main-sequence; (2) the critical mass ratio of a more massive donor star is smaller than a less massive star; (3) the critical mass ratio of low mass main sequence stars increases with mass from 0.63 to $1.6 M_{\odot} ;(4)$ the critical mass ratio of low mass main sequence donor stars with mass less than about $0.5 M_{\odot}$ is nearly $2 / 3$.

We give the fitting formula of the critical mass ratio for the donor stars on the main sequence. The critical mass ratio of the main sequence donor stars seems to be linear with the common logarithm of the mass and radius (formula 3.1),

$$
q_{\text {crit }}=c_{1}+c_{2} \lg M / M_{\odot}+c_{3} \lg R / R_{\odot} .
$$

The coefficients of the fitting formula are 2, 1.43 and 4.03 for MS donor stars with mass from 0.63 to $1.6 M_{\odot}$, and are $2.93,-3.62$ and 4.23 for donor stars with mass from 1.6 to $100 M_{\odot}$. The relative error of the fitting results is less than $20 \%$.

\section{References}

Ge, H., Webbink, R. F., Chen, X., \& Han, Z. 2010, ApJ, 717, 724 